\title{
EL ÍNDICE DE INSEGURIDAD LINGÜÍSTICA EN LA RADIO LOCAL ALMERIENSE
}

\begin{abstract}
López González Antonio Maria, El indice de inseguridad lingüistica en la radio local almeriense [The linguistic confidence rate in the local Almeria radio]. Studia Romanica Posnaniensia, Adam Mickiewicz University Press, Poznań, vol. XXXII : 2005, pp. 57-64. ISBN 83-232-1465-4, ISSN $0137-2475$.
\end{abstract}

The linguistic confidence rate provides us with information about the level of convergence or divergence between the sociolinguistic consciousness and the performance inside a spech community. This causes the stabilization of the linguistic varieties, when the coincidence occtrs, or the linguistic change, in the case of discrepancy. For the analysis of the linguistic confidence, it is necessary to compare the attitude to the linguistic performance. This phenomenon is what this article aims at: To show and to analyze the correlation between the journalists' sociolinguistic attitude and the performance during the daily local broadcasting in Almeria (Spain).

Son escasos los estudios acerca de la inseguridad lingüistica. Entre unos pocos investigadores, sólo Labov (1966: 464; 1972: 162, 178-179) en Nueva York, López Morales (1979: 168-169) en Puerto Rico, y García Marcos (1990: 126-127) en la Costa Granadina se han ocupado del análisis de este índice sociolingüístico. Y eso, a pesar de que "el grado de congruencia entre lo que el hablante piensa - y en consecuencia, se supone que prefiere o rechaza a priori-y lo que en realidad lleva a la práctica, desde el punto de vista variacionista, proporciona datos sumamente valiosos acerca de la vitalidad de los fenómenos lingüísticos observados" (García Marcos, 1999: 171).

Para el análisis de la inseguridad lingüística es preciso confrontar los datos de las actitudes con las actuaciones lingüísticas. Esto es to hacemos en el presente artículo. Presentamos la correlación existente entre las actitudes sociolingüísticas de los profesionales de los medios de comunicación de Almería (España) y sus actuaciones en la práctica radiofónica local, y analizamos la correspondencia que se da entre las actuaciones de los informadores y sus actitudes. Para ello ponemos en relación los resultados relativos al grupo de los profesionales de los medios de información en el estudio de García Marcos y Fuentes González (1996) con los 
datos obtenidos en López González (2001). De esta manera, comprobamos la coincidencia o discrepancia entre la conciencia sociolingǘstica y la actuación en este grupo de prestigio de la comunidad de habla de Almeria, lo que lleva a la estabilización de las variedades linguísticas, en el caso de la coincidencia, o a al cambio lingüístico, de darse la discrepancia.

Hemos de aclarar aquí que el trabajo de García Marcos y Fuentes González (1996) citado aborda el estudio de un grupo social mucho más amplio, el compuesto por los estratos de prestigio de la sociedad almeriense, que incluye a religiosos, profesores (de enseñanza universitaria, de enseñanza media y de enseñanza primaria), juristas e informadores. En este punto nos limitamos solamente a un componente de este grupo, los informadores. No obstante, el interés de los datos de inseguridad lingüística justifica esta relativa heterogeneidad en la base de confrontación.

Medimos el nivel de convergencia (seguridad) o divergencia (inseguridad) entre las actuaciones linguísticas y las actitudes hacia ellas a partir de la diferencia entre ambas magnitudes (Actuación - Actitud de apoyo). A medida que se aproxima a cero mayor es la seguridad; si se desvía $(+/-)$ de cero, es un indice de inseguridad sociolingüistica, tanto mayor cuanto mayor sea la desviación.

Cuadro I

Actitudes sociolingüisticas de los informadores en Almeria'

\begin{tabular}{|c|c|c|c|c|}
\hline $\mathrm{V}$ & + & - & $=$ & 1 \\
\hline Pérdida de $/-\mathrm{s} /$ & 32,49 & 36,47 & 24,12 & 6,74 \\
\hline $\mid 1 />/ \mathrm{r} /$ & 4,82 & 54,14 & 28,11 & 12,44 \\
\hline $\mid-\mathrm{r}+\mathrm{lo} />/-\varnothing+\mathrm{l}_{0} /$ & 2,74 & 86,25 & 9,53 & 1,05 \\
\hline Ceceo & 2,26 & 87,64 & 8,19 & 1,45 \\
\hline /a/ protética & - & 81,74 & - & 9,25 \\
\hline$|-d-1>| \varnothing \mid$ & 3,26 & 83,28 & 5,17 & 7,28 \\
\hline "Se me" > "me se" & - & 83,16 & 2,32 & 14,51 \\
\hline Usos locativos del posesivo & 41,45 & 26,42 & 10,54 & 20,08 \\
\hline Dequeísmo & 65,15 & 8,74 & 5,62 & 19,37 \\
\hline "Más nada" & 79,18 & 17,42 & - & 2,21 \\
\hline Infinitivo como imperativo & 46,56 & 23,48 & 15,37 & 13,76 \\
\hline Futuros perifrásticos & 97,76 & - & - & 3,62 \\
\hline "os" > "sus" & - & 100,00 & - & - \\
\hline "En base a" & 92,48 & 2,37 & 5,14 & - \\
\hline "Cuanto" > "contra" & - & 83,47 & 16,52 & - \\
\hline Lcísmo & 92,32 & 5,44 & - & 2,23 \\
\hline Sufijo $-i c o$ & 51,25 & 12,42 & 36,32 & - \\
\hline "Como" superfluo & 46,29 & - & - & 54,70 \\
\hline "El hecho de que" & 70,84 & - & - & 29,16 \\
\hline -ATA & - & 88,52 & 11,47 & - \\
\hline
\end{tabular}

'Tomado de Garcia Marcos y Fuentes González (1996: 82-87). 
La primera de las variantes que sometemos a consideración es la pérdida de la /-s/ implosiva. Ésta era apoyada por un tercio de los encuestados en el estudio de García Marcos y Fuentes González (1996), en tanto que un $4 \%$ más estaba en contra. En López González (2001), la pérdida de /-s/ implosiva por parte de los locutores radiofónicos ha supuesto solamente el $7,07 \%$, lo que se traduce en la actuación en un uso cinco veces menor al apoyo recibido por esta variante. Los locutores han potenciado la variante centro-peninsular, optando por la conservación de la /-s/ implosiva casi en un $80 \%$. La otra variante en juego obtuvo en la actuación $\tan$ solo un $13 \%$ de las ocurrencias. De esto se deduce que la actuación en relación con esta variable aún es más conservadora que la actitud mostrada hacia ésta. En la actuación se agudiza la tendencia negativa hacia esta variante, previamente mostrada en su juicio sociolingüistico, lo que denota una alta inseguridad lingüística $(-25,42 \%)$.

En el estudio de actitudes sociolingüisticas, el rotacismo de $/ 1 />/ \mathrm{r} /$ fue fuertemente censurado. En la muestra analizada en López González (2001), tan sólo una vez está presente este fenómeno en un locutor, y éste realiza el cambio de $/ 1 /$ por $/ \mathbf{r} /$ cuando, al hablar con un radioyente, éste también lo hace. En todo caso, se trata de un único caso. El comportamiento lingüístico de los locutores con respecto a esta variante es totalmente normativo. La seguridad lingüística es elevada $(-4,82 \%)$

El debilitamiento y pérdida de /-r/ en el infinitivo ante los pronombres enclíticos personales de tercera persona, altamente censurado por los periodistas en sus actitudes sociolingüisticas, ha seguido en la actuación un camino similar de apego al español estándar. Sin embargo, en la actuación se pierde esta $/-\mathbf{r} /$ en un $11,11 \%$, lo que implica un grado mayor de uso en la práctica que lo que hacía suponer el exiguo $2,74 \%$ de apoyo cosechado por esta variante entre los profesionales de los medios de comunicación. Se apunta, por consiguiente, un grado importante de inseguridad $(+8,26 \%)$.

Tanto en el juicio de la variante como en el uso que de ésta se efectúa en la práctica, el ceceo se halla estigmatizado por los comunicadores, coincidiendo, por tanto, juicio y actuación lingüística.

La /a/ protética, que sí aparece en hablantes no locutores profesionales, está totalmente ausente de las actuaciones lingüísticas de los locutores profesionales. Por tanto, se da una absoluta coincidencia entre actuación y actitud: es en la muestra un ejemplo claro de seguridad sociolingüística.

La pérdida de la $/-\mathrm{d}-/$ intervocálica en participios, tan arraigada en el lenguaje cotidiano de la calle, coloquial, e incluso en entrevistas a personajes públicos en Almería, se halla prácticamente desterrada del lenguaje de los locutores de radio. En ellos se da, por consiguiente, un alto índice de seguridad lingüística $(+1,44 \%)$, ya que éstos también muestran su rechazo por la pérdida de esta /-d-/ en participios. 
Índices de seguridad / inseguridad lingüística en la radio local almeriense

\begin{tabular}{|c|c|c|c|}
\hline Variante & Actuación (\%) & Aclitud (\%) & Seguridad/Inseguridad $(\%)$ \\
\hline Pérdida de $/$-s/ & 7,07 & 32,49 & $-25,42$ \\
\hline$|-\mathrm{r}+\mathrm{lo} />|-0+1 \mathrm{o} \mid$ & 11,11 & 2.74 & $+8,26$ \\
\hline$|-\mathrm{d}-|>|\varnothing|$ & 4,70 & 3,26 & +1.44 \\
\hline Futuro perifrástico & 46,10 & 97,76 & $-51,66$ \\
\hline Sufijo-ico & 8,54 & 51,25 & 42,71 \\
\hline$n />/ \mathrm{r} /$ & 0,00 & 4,82 & $-4,82$ \\
\hline Ceceo & $* *^{2}$ & 2,26 & $(\approx$ Seguridad $)$ \\
\hline /a/protética & 0,00 & 0,00 & 0,00 \\
\hline "Se me" > "me se" & 0.00 & 0,00 & 0,00 \\
\hline Usos locativos del posesivo & 0,00 & 41,45 & $-41,45$ \\
\hline "Más nada" & 0,00 & 79,18 & 79,18 \\
\hline Dequeísmo & 0,00 & 65,15 & -65.15 \\
\hline "os" > "sus" & 0.00 & 0,00 & 0,00 \\
\hline "en base a" & 0,00 & 92,48 & $. .92,48$ \\
\hline "cuanto" > "contra" & 0,00 & 0,00 & 0,00 \\
\hline Leísmo & 3,33 & 92.32 & $-88,99$ \\
\hline "el hecho de que" & 0,00 & 70,84 & $-70,84$ \\
\hline$-a t a$ & 0,00 & 0,00 & 0,00 \\
\hline "como" superfluo & 0,00 & 46,29 & $-46,29$ \\
\hline Infinitivo $>$ Imperativo & Tres casos & 46,56 & .- \\
\hline
\end{tabular}

La inversión sintáctica de "se me" por "me se", los usos locativos del posesivo y la utilización de "más nada" en lugar de "nada más" han carecido de ocurrencias en la muestra que estudiamos en López González (2001). Esto no puede sorprender en el caso de la inversión "se me" por "me se", puesto que el apoyo que esta variante obtuvo en el estudio de las actitudes sociolingüísticas fue nulo. De ello se deduce una seguridad lingüística absoluta entre los profesionales de los medios de comunicación en relación con esta variable. Más sorprendente es, en cambio, la ausencia de usos locativos del posesivo y de la expresión "más nada", que, por el contrario, obtuvo un gran apoyo entre los encuestados. Aquí, en conclusión, se presenta una situación discordante entre la actitud hacia estas variantes y el comportamiento sociolingüístico. Hablamos en estos casos, por tanto, de unos indices de inseguridad lingüística elevadísimos, $-41,45 \%$ para los usos locativos del posesivo y $-79,18 \%$ para la expresión "más nada".

El tan denostado dequeismo de los medios, que presentaba una corriente favorable entre los locutores, curiosamente carece de ocurrencias entre ellos, apareciendo solamente en el grupo de los no locutores. Acaso estemos, en relación con el

\footnotetext{
${ }^{2}$ Sólo un locutor, esporádicamente, en conversación con radioyente ceccantc.
} 
llamado "dequeísmo", en una situación de especial cuidado de los locutores y de uso de la norma, tras las muchas críticas de las que han sido objeto, en contra de su propia actitud positiva hacia este fenómeno. La inseguridad, por tanto, es muy elevada: $-65,15 \%$.

La actitud favorable que manifiestan los profesionales de los medios de comunicación hacia el uso del infinitivo con valor de imperativo tiene su correlato positivo en la actuación lingüística de éstos. Así aparecen casos de infinitivo como imperativo, tanto afirmativo como negativo, en la Cadena COPE y la Cadena 100 , y siempre por parte de los locutores.

El abrumador apoyo a las formas perifrásticas para la expresión del futuro que mostraban los periodistas encuestados por García Marcos y Fuentes González (1996), desciende en la práctica ante los micrófonos, ya que, si bien las ocurrencias de las formas analíticas en nuestra muestra representaban el $46,10 \%$ del total de formas de futuro, las formas sintéticas son, en definitiva, las más usadas, con un $53,90 \%$ del total. Se da, en principio, una situación de inseguridad lingüística en relación con los futuros perifrásticos $(-51,66 \%)$. En cualquier caso, el porcentaje de formas perifrásticas es lo suficientemente alto como para pensar, que la actitud positiva hacia esta variante se ve reflejada en la actuación lingüística, puesto que una actitud positiva hacia una variante no tiene porque suponer automáticamente un rechazo a otras variantes del espectro.

La tríada formada por la sustitución de la forma "os" por el posesivo "sus", la expresión "en base a" y la utilización de la preposición "contra" por "cuanto" no obtuvo ocurrencia alguna en la muestra recogida en López. González (2001). En el caso de "sus" cn lugar de "os" y en la sustitución de "cuanto" por "contra" se trata de un ficl reflejo en la práctica lingüística del rechazo frontal en las actitudes a estas variantes, lo que demuestra una seguridad lingüística total. Diferente es el caso de "en base a", favorecido por los periodistas, pero, como decimos, sin ocurrencias en la muestra analizada.

No existe coincidencia entre la positiva actitud de los informadores de los medios de comunicación hacia el leísmo y la escasísima frecuencia de este leísmo en la radio local almeriense. Tan sólo en dos ocasiones los locutores radiofónicos utilizaron en las grabaciones realizadas el pronombre "le" como objeto directo. El indice de inseguridad es muy alto, un $88,99 \%$. Frente a este hecho, hubo un uso mayoritariamente normativo de distinción de "le" / "lo" en la muestra analizada. Ello demuestra la pervivencia de la distinción en Almería, aunque los casos aparecidos, aún siendo testimoniales, y la positiva actitud hacia el fenómeno, denotan una tendencia a la aceptación del leísmo.

Con ser el sufijo -ico la variante dialectal de la zona para la formación del diminutivo, y a pesar de contar con una actitud positiva de los periodistas, no obtiene un apoyo similar en las actuaciones de los informadores. De hecho, entre estos, es la variante menos representada, con un $8,54 \%$, que contrasta con el $51,25 \%$ de opinión favorable entre estos mismos. Esto supone una notable discrepancia entre lo 
que las actitudes preveían y lo que la actuación indica. La inseguridad lingüística es alta: $-42,71 \%$.

Finalmente, el último grupo de variantes que rastreamos en nuestra muestra, el uso de "como" superfluo, la expresión "el hecho de que" y el sufijo -ata no contaron, una vez más, como algunas de las variantes que aquí sometemos a análisis, con ocurrencia alguna. Con respecto al sufijo -ata, la no-actualización en la muestra de éste era más que previsible, si se tiene en cuenta que el juicio dispensado acerca de esta variante fue de rechazo total. La seguridad es, por tanto, máxima. Distinto es el caso de "como" innecesario y de la expresión "el hecho de que", que en las encuestas a los informadores fueron valorados positivamente, y que jamás son empleados en nuestras grabaciones. La situación aquí es inversa y marca, en consecuencia, una alta inseguridad sociolingüistica.

Concluimos. Deciamos al principio que el estudio de la inseguridad linguística proporcionaba indicios importantes acerca de la estabilización de las variedades lingüísticas o del cambio lingüístico, según el caso. No cabe duda de que los profesionales de los medios de comunicación, con sus actuaciones lingüísticas y sus actitudes- contribuyen, gracias a su protagonismo social, a ambos procesos. $Y$ en este estudio ha quedado patente que el grado de inseguridad lingüística hacia los diferentes fenómenos y variantes lingüísticas tomadas en consideración ha puesto de manifiesto estos procesos de estabilización o de cambio en la práctica radiofónica local de Almeria.

Así, de una parte, se observa un grupo de variantes en las que actitudes y actuación convergen en un $100 \%$, tanto en su rechazo como en su no actualización en el discurso. Estas variantes han sido tradicionalmente calificadas como vulgares. Entre ellas se cuenta la $a$ - protética, el cambio de orden se me $>$ me se y las sustituciones os > sus y cuanto > contra. Igualmente pertenece a este grupo la sufijación en -ata, propia de la jerga juvenil, según Casado Velarde (1988: 102-108). El indice de seguridad ha sido también muy alto con relación al ceceo y a la elisión de /-d-/ en participios. La inseguridad ronda tan sólo el $1 \%$ en ambos casos. Tanto el ceceo, propio de importantes zonas de Andalucía (Lapesa, 1957: 67-94), como la elisión de /-d-/ en participios, muy extendida geográfica y socialmente en todas las hablas hispánicas (Alcina y Blecua, $1988^{6}: 328$ ) y caracteristica del dialecto andaluz, segín Salvador (1964: 183-188), están estigmatizados por los sectores cultos de la sociedad como vulgarismos (Navarro Tomás, 1982²1: 101, 109; Alarcos Llorach, $1983^{4}: 185$; Marsá, 1986: 30). Por lo tanto, con respecto a estas variantes se apunta una situación lingüistica sólida en el medio radiofónico almeriense.

Mayor indecisión denota el índice de inseguridad lingüística con respecto a la neutralización de $-1 /-r$ implosivas $(-4,82 \%)$ y al debilitamiento y pérdida de $/-\mathrm{r} /$ final de infinitivo seguida de pronombre, $/-\mathrm{r}+1 \mathrm{o} /(8,26 \%)$. Se trata de rasgos peculiares de la fonética andaluza, sobre todo en el caso de la deslateralización de /-1/ (Salvador, 1964: 183-188, Alcina y Blecua, $1988^{6}: 365$ ), pero estigmatizados sociolingüistica 
y normativamente (Vaz de Soto, 1981; Carbonero, 1985; García Marcos, 1992: 50), si bien esta estigmatización no es tan fuerte en el caso de $/-\mathrm{r}+\mathrm{lo} /$.

La inseguridad lingüística, con valores negativos superiores al $-25 \%$, surge en relación con fenómenos y variantes apoyadas en las actitudes en todos los casos, típicos del andaluz como la pérdida de $/$-s/ implosiva $(-25,42 \%$ ) (Salvador, 1964: 183-188; Vaz de Soto, 1981; Zamora Vicente, 1967), o peculiares de la Andalucía Oriental como el diminutivo en -ico ( $-42,71 \%)$ (Alvar, 1959). Aparece también en fenómenos muy frecuentes en el español de nuestros dias, como los usos del infinitivo con valor imperativo (Lorenzo, 1971: 104; Alcina y Blecua, 19886: 747), los usos locativos del pronombre posesivo $(-41,45 \%)$ (Alcina y Blecua, $1988^{6}: 619$ 620 ), el leismo, con fuerte arraigo en el español centro-peninsular $(88,99 \%$ ) (Marsá, 1986: 138-139; De los Mozos, 1984: 19). Del mismo modo, el futuro perifrástico "ir a + infinitivo", que protagoniza uno de los cambios más destacados del español actual (Bauhr, 1989; Berschin, 1987; Berschin, Fernández-Sevilla y Felixberger, 1987; García Marcos, 1991; Lamíquiz y Carbonero, 1987; Moreno de Alba, 1978; Paufler, 1970), alcanza una inseguridad lingüistica del 51,66\%. Igualmente obtienen altos porcentajes de inseguridad el dequeísmo $(65,15 \%)$, las locuciones en base a $(92,48 \%)$ y el hecho de que $(70,84 \%)$, la modificación en el orden secuencial de más nada $(79,18 \%)$ y el uso con valor aproximativo de "como" $(46,29 \%)$, todos cllos promovidos entre sectores con prurito cultista o por los medios de comunicación (Marsá, 1986: 114, 154-156). En consecuencia, la situación lingüística en la radio local almeriense en relación con este grupo de variantes es sumamente inestable y denota, por eso, una situación de cambio lingüístico.

\section{BIBLIOGRAFIA}

Alarcos Llorach E. (1983), Fonologia española, Gredos, Madrid.

Alcina Franch J., Blecua J.M. (1975), Gramática española, 6a ed., 1988. Ariel, Barcelona.

Alvar M. (en colaboración con A. Llorente y G. Salvador) (1959), Atlas Lingüistico y Etnográfico de Andalucia (A.L.E.A.), C.S.I.C., Granada.

Baubr G. (1989), El futuro en -ré é ir a + infinitivo en español peninsular moderno, Acta Universitatis Gothoburgensis (Románica Gothoburgensia), Gotemburgo.

Berschin H. (1987), Futuro analitico y futuro sintético en el español peninsular y colombiano, Lingüística Española Actual, IX, I, Madrid, Instituto de Cooperación Iberoamericana, pp. 101. 110.

-, Fernández-Sevilla J., Felixberger J. (1987), Die Spanische Sprache, Hueber, Munich.

Carbonero Ca no P. (1985), Norma estándar y actitud sociolingüistica. Sobre la aceptación y uso de algunos rasgos lingüísticos en hablantes sevillanos, Sociolingüística andaluza, I. Servicio de Publicaciones de la Universidad de Sevilla, Sevilla.

Casado Velarde M. (1988), Lenguaje y cultura, Sintesis. Madrid.

De Los Mozos S. (1984), La norma castellana del español, Ámbito, Valladolid. 
García Marcos F.J. (1991), Estratificación social del español de la costa granadina. Depto. de Lingüistica General y Teoría de la Literatura, Almería.

-, (1992), Estratificación social de -ir/ en el español de la Costa Granadina, Anuario de Letras, XXX, pp. 45-79.

-, (1999), Fundamentos críticos de sociolingüistica. Almeria, Servicio de Publicaciones de la Universidad de Almeria.

-, Fuentes González A.D. (1996), Mecanismos de prestigio y repercusión sociolingüistica. Estratifícación social del espanol de Almeria, III, Grusta, Almeria.

Labov W. (1966). The Social Stratification of English in New York City, Center for Applied Linguistics, Washington.

-, (1972), Modelos sociolingüisticos, Cátedra, Madrid 1983.

Lamiquiz Ibáñez. V., Pedro Carbonero (1987), Perfil sociolingüistico del sevillano culto. Instituto de Desarrollo Regional, Sevilla.

Lapesa R. (1957), Sobre el seseo y el ceceo andaluces en Miscelánea Homenaje a André Martinet (estructuralismo e historia), I. Universidad de La Laguna, pp. 67-94.

López González A.M. (2001), El lenguaje radiofónico de la ciudad de Almeria. Análisis sociolingïistico, Universidad de Almeria. Tesis doctoral inédita, Almería.

López Morales H. (1979), Indices de inseguridad lingüistica en San Juan en Dialectologia y sociolingüistica. Temas puertorriqueños, Hispanova, Madrid, pp. 173-185.

Lorenzo E. (1971), El español de hoy, lengua en ebullición, Gredos, Madrid.

Marsá F. (1986), Diccionario normativo y guía práctica de la lengua española, Ariel, Barcelona.

Moreno De Alba G. (1978), Valores de las formas verbales en el español de México, UNAM, México.

Navarro Tomás T. (1982 21 , Manual de pronunciación española, C.S.I.C./R.F.E., Madrid.

Paufler H. (1970), Das periphrastische Futur im Kubanischen Spanisch, Beiträge zur Romanischem Philologie, 9, págs. 135-137.

Salvador G. (1964), La fonética andaluza y su propagación social y geográfica en Presente y futuro de la lengua española, II, O.F.I.N.E.S., Madrid, pp. 183-188.

Vaz de Soto J.M. (1981), Defensa del habla andaluza, Edisur, Sevilla.

Zamora Vicente A. (1967), Dialectología española, Gredos, Madrid. 